\title{
COMPLETE LINEAR SYSTEMS \\ ON RATIONAL SURFACES
}

BY

BRIAN HARBOURNE

\begin{abstract}
We determine the dimension, fixed components and base points of complete linear systems on blowings-up of $\mathbf{P}^{2}$ having irreducible anticanonical divisor.
\end{abstract}

Consider a set of points $p_{1}, \ldots, p_{n}$ of the projective plane $\mathbf{P}_{k}^{2}$. The ground field $k$, fixed throughout this paper, is algebraically closed of arbitrary characteristic. We allow $p_{j}$ to be infinitely near $p_{i}$ for $j>i$. One can ask the following questions: What is the dimension of the linear system of curves of degree $d$ passing through each point $p_{i}, i=1, \ldots, n$, with multiplicity $m_{i}$ ? Assuming that the linear system is not empty, what are its fixed components and base points?

The answers, of course, depend on the points. In [7, §5], Nagata gives a partial answer to the first question, in case $n \leqslant 9$ and the points $p_{i}$ are not too special. However, even in the case that the points are independent generic points, these questions remain unanswered in general (see, for example, [8]). In this paper we answer these questions under the condition that the points $p_{1}, \ldots, p_{n}$ are nonsingular points of an irreducible, reduced cubic curve in $\mathbf{P}^{2}$.

We take the following approach. Studying linear systems with assigned base points $p_{i}$ is equivalent to studying complete linear systems (i.e., the global sections of divisor classes) on the surface $V$ obtained by blowing up the points $p_{i}$ [4, p. 395]. Demazure studies such surfaces in [1], mostly assuming that $n \leqslant 8$. The requirement that these points lie on a reduced, irreducible cubic becomes the condition that, if $K$ is the canonical class of $V,-K$ has an irreducible, reduced section $D$. This situation is similar to that of $[5, \S 1]$ in which Looijenga determines the effective divisor classes and the irreducible components for exceptional curves; indeed, our work is largely motivated by these results.

In this paper we study divisor classes (divisors modulo rational equivalence) on such surfaces $V$. We define a certain class of divisor classes, the almost excellent divisor classes (cf. Corollary 3.2). In Theorem 1.1 and Proposition 1.2 of $\S 1$ we compute the dimension $h^{0}(V, \mathscr{F})$ of global sections of $\mathscr{F}$ for any almost excellent class $\mathscr{F}$.

In Theorem 2.1 of $\S 2$ we show that, for any class $\mathscr{F}$, either $h^{0}(V, \mathscr{F})=0$ or $\mathscr{F}=\mathscr{G}+\mathscr{C}$, where $\mathscr{G}$ and $\mathscr{C}$ are the classes of effective divisors, $h^{0}(V, \mathscr{G})=h^{0}(V, \mathscr{F})$,

Received by the editors April 16, 1984.

1980 Mathematics Subject Classification. Primary 14C20.

01985 American Mathematical Society $0002-9947 / 85 \$ 1.00+\$ .25$ per page 
and $\mathscr{G}$ is almost excellent. Our proof is constructive, and only depends upon knowing the kernel of the homomorphism $j^{*}$ : Pic $V \rightarrow$ Pic $D$ of the groups of divisor classes induced by the inclusion $j: D \subset V$.

In particular, this gives an algorithm for computing $h^{0}(V, \mathscr{F})$ for any divisor class $\mathscr{F}$ on $V$. As an application, we determine in Theorem 3.1 and Corollary 3.4 of $\S 3$ those complete linear systems on $V$ having fixed components or base points, and we determine those fixed components and base points.

Part of this paper comprises part of our thesis, written under the direction of $\mathbf{M}$. Artin, to whom we express our appreciation.

0. Preliminaries. To be precise, we consider an algebraic surface $V$ for which we have a sequence of morphisms:

$$
V=V_{n} \stackrel{\pi_{n}}{\rightarrow} V_{n-1} \stackrel{\pi_{n-1}}{\rightarrow} \cdots \stackrel{\pi_{2}}{\rightarrow} V_{1} \stackrel{\pi_{1}}{\rightarrow} V_{0}=\mathbf{P}^{2}
$$

Each morphism $\pi_{i}$ is the blowing-up of $V_{i-1}$ at a point $p_{i}$. Since $\pi_{i}$ induces an inclusion $\pi_{i}^{*}$ : Pic $V_{i-1} \rightarrow \operatorname{Pic} V_{i}$, we will find it convenient to regard Pic $V_{i}$ as a subgroup of Pic $V, i=0, \ldots, n$. With respect to this abuse of notation, which we justify in Lemma 1.3, it is well known [4, V.3] that Pic $V_{i}$ is the free abelian group generated by the class $\mathscr{E}_{0}$ of a line in $\mathbf{P}^{2}$ and the classes $\mathscr{E}_{j}, j=1, \ldots, i$, where $\mathscr{E}_{j}$ is the class of the exceptional divisor $E_{j}=\pi_{j}^{-1}\left(p_{j}\right)$.

Modifying slightly the usage of [5] (cf. [1]), we call such a collection $\mathscr{E}=$ $\left\{\mathscr{E}_{0}, \ldots, \mathscr{E}_{n}\right\}$ of divisor classes of $V$ an exceptional configuration for $V$. We remark that the sequence of morphisms of $(0.1)$ can be recovered from the exceptional configuration $\mathscr{E}$, since $\pi_{i}$ is simply the contraction of the unique global section $E_{i}$ of $\mathscr{E}_{i}$ on $V_{i}[4, \mathrm{~V} .3]$. Thus there is a bijective correspondence between exceptional configurations for $V$ and such sequences of morphisms $(0.1)$.

The canonical class $K$ of $V$ takes the form $-3 \mathscr{E}_{0}+\mathscr{E}_{1}+\cdots+\mathscr{E}_{n}$ for any exceptional configuration $\mathscr{E}=\left\{\mathscr{E}_{0}, \ldots, \mathscr{E}_{n}\right\}[4$, V.3]. With respect to a given exceptional configuration, and hence a given sequence of morphisms $(0.1)$ being understood, we denote by $K_{i}$ the canonical class of $V_{i}$.

For the rest of this paper we assume $-K$ has an irreducible, reduced section. We fix a choice of one, and call it $D$. Of course, the image of $D$ under the morphism $V \rightarrow V_{i}$ is isomorphic to $D$, and is a section of $-K_{i}$ on $V_{i}$. No confusion should result if we refer to it also as $D$. The inclusion $j: D \subset V$ induces the mapping $j^{*}$ : Pic $V \rightarrow$ Pic $D$ of Picard groups.

We will need to make use of the intersection product on Pic $V$. With respect to an exceptional cnfiguration $\left\{\mathscr{E}_{0}, \ldots, \mathscr{E}_{n}\right\}$, this product is defined by being bilinear and symmetric, $\mathscr{E}_{i} \cdot \mathscr{E}_{j}=0, i \neq j, \mathscr{E}_{0}^{2}=1, \mathscr{E}_{i}^{2}=-1, i>0[4, \mathrm{~V} .3]$.

We also need to recall the formula of Riemann-Roch for smooth, complete, rational surfaces. If $X$ is such a surface, then, for any divisor class $\mathscr{F}$ on $X$ [4],

(0.2) $\quad h^{0}(X, \mathscr{F})-h^{1}(X, \mathscr{F})+h^{2}(X, \mathscr{F})=\frac{1}{2}(\mathscr{F} \cdot \mathscr{F}-\mathscr{F} \cdot K)+1$.

If $\left\{\mathscr{E}_{0}, \ldots, \mathscr{E}_{n}\right\}$ is an exceptional configuration on $X$, we have the easy facts [1, p. 24] that

$$
\begin{array}{ll}
\text { (a) } \quad h^{0}(X, \mathscr{F})=0 & \text { if } \mathscr{F} \cdot \mathscr{E}_{0}<0, \\
\text { (b) } \quad h^{2}(X, \mathscr{F})=0 & \text { if } \mathscr{F} \cdot \mathscr{E}_{0} \geqslant-2 .
\end{array}
$$


We will also use [1, pp. 51 and 59]:

Let $F$ be an irreducible reduced divisor on $V$, having divisor class $\mathscr{F}$. Let $\mathscr{G} \in$ Pic $V$ and define

$$
g(F)=\frac{1}{2}(\mathscr{F} \cdot \mathscr{F}+K \cdot \mathscr{F})+1 .
$$

If $\mathscr{F} \cdot \mathscr{G} \geqslant 2 g-1$, then $h^{1}\left(F, \mathscr{G} \otimes \mathcal{O}_{F}\right)=0$. If $h^{1}(V, \mathscr{G}-\mathscr{F})$ $=0$ and $\mathscr{F} \cdot \mathscr{G} \geqslant 2 \mathrm{~g}$, then $\mathscr{G}$ is effective and generated by global sections at the points of $F$.

Our results are stated using the following terminology. Let $\mathscr{E}=\left\{\mathscr{E}_{0}, \ldots, \mathscr{E}_{n}\right\}$ be an exceptional configuration for $V$, and denote by $r_{i}, i=1, \ldots, n-1$, the class $\mathscr{E}_{i}-$ $\mathscr{E}_{i+1} ;$ also, $r_{-2}=\mathscr{E}_{0}-\mathscr{E}_{1}, r_{-1}=\mathscr{E}_{0}-\mathscr{E}_{1}-\mathscr{E}_{2}$ and $r_{0}=\mathscr{E}_{0}-\mathscr{E}_{1}-\mathscr{E}_{2}-\mathscr{E}_{3}$. The classes $r_{0}, \ldots, r_{n-1}$ are the simple roots of a root system in Pic $V$ [5]. We call a class $\mathscr{F} \in \operatorname{Pic} V$

$\mathscr{E}$-standard if $\mathscr{F} \cdot r_{i} \geqslant 0, i \geqslant-2$, and $\mathscr{F} \cdot \mathscr{E}_{i} \geqslant 0, i \geqslant 0$;

almost $\mathscr{E}$-excellent if $\mathscr{F}$ is $\mathscr{E}$-standard and $\mathscr{F} \cdot K \leqslant 0$; and

$\mathscr{E}$-excellent if $\mathscr{F}$ is almost $\mathscr{E}$-excellent and $\mathscr{F} \cdot K<0$.

We refer to a class $\mathscr{F}$ as being standard (almost excellent, or excellent resp.) if there is an exceptional configuration $\mathscr{E}$ for which $\mathscr{F}$ is $\mathscr{E}$-standard (etc.). We will also find it convenient to refer to $\mathscr{F}$ as being effective if $h^{0}(V, \mathscr{F})>0$ and irreducible if $\mathscr{F}$ has a reduced and irreducible section. Moreover, if $\mathscr{F}$ is the divisor class of an effective divisor $F$, we will refer to the class $\mathscr{H}$ of a fixed component $H$ of the linear system $|F|$ as a fixed component of $\mathscr{F}$. Similarly, we will refer to a base point of $|F|$ as being a base point of $\mathscr{F}$.

1. Dimension of sections of almost excellent classes. Our main results of this section are the two that follow.

THEOREM 1.1. Let $\mathscr{F}$ be an element of Pic $V$.

(a) If $\mathscr{F}$ is standard, then $\mathscr{F}$ is effective.

(b) If $\mathscr{F}$ is excellent, then $h^{0}(V, \mathscr{F})=\frac{1}{2}(\mathscr{F} \cdot \mathscr{F}-\mathscr{F} \cdot K)+1$ and $h^{1}(V, \mathscr{F})=$ $h^{2}(V, \mathscr{F})=0$.

PROPOSITION 1.2. Let $\mathscr{F}=a_{0} \mathscr{E}_{0}+a_{1} \mathscr{E}_{1}+\cdots+a_{n} \mathscr{E}_{n}$ be an almost $\mathscr{E}$-excellent class for an exceptional configuration $\mathscr{E}=\left\{\mathscr{E}_{0}, \ldots, \mathscr{E}_{n}\right\}$, and suppose that $\mathscr{F} \cdot K=0$. Then one of the two cases that follow must occur:

(a) $\mathscr{F}=-m K_{9}$ for some $m \geqslant 0$, or

(b) $\mathscr{F}^{\prime}=\left(a_{0}-3\right) \mathscr{E}_{0}+\sum_{i>0 ; a_{i} \leqslant-1}\left(a_{i}+1\right) \mathscr{E}_{i}$ is $\mathscr{E}$-excellent.

Moreover, in case (a), $h^{0}(V, \mathscr{F})=[m / l]+1$, where lis the order of $j^{*}\left(-K_{9}\right)$ in Pic $D$ and $[\cdot]$ is the greatest integer function, while for case (b) we have

$$
h^{0}(V, \mathscr{F})= \begin{cases}\frac{1}{2}\left(\mathscr{F}^{\prime} \cdot \mathscr{F}^{\prime}-\mathscr{F}^{\prime} \cdot K\right)+1 & \text { if } j^{*}(\mathscr{F}) \neq 0, \\ \frac{1}{2}\left(\mathscr{F}^{\prime} \cdot \mathscr{F}^{\prime}-\mathscr{F}^{\prime} \cdot K\right)+2 & \text { if } j^{*}(\mathscr{F})=0 .\end{cases}
$$

Before we give the proofs we consider a series of lemmas, beginning with

LEMMA 1.3. Let $S^{\prime} \stackrel{\pi}{\rightarrow} S$ be the blowing-up of a smooth surface $S$ at a point $p$, and let $\pi^{*}$ : Pic $S \hookrightarrow \operatorname{Pic} S^{\prime}$ be the induced map. Then (1) $\pi^{*}$ preserves cohomology and (2) a 
line bundle $\mathscr{F}$ is generated by global sections at a point $q \in S$ iff $\pi * \mathscr{F}$ is generated by global sections along $\pi^{-1}(q)$.

Proof. Let $\mathscr{F} \in$ Pic $S$ and let $\mathscr{F}^{\prime}=\pi^{*} \mathscr{F}$. Then $\pi_{*} \mathscr{F}^{\prime}=\mathscr{F} \otimes \pi_{*} \mathcal{O}_{S^{\prime}}$, but $\pi_{*} \mathcal{O}_{S^{\prime}} \simeq$ $\mathcal{O}_{S}$ and $R^{i} \pi_{*} \mathscr{F}^{\prime}=0$ for $i>0\left[4\right.$, p. 387]. Therefore, $H^{j}(S, \mathscr{F}) \cong H^{j}\left(S^{\prime}, \mathscr{F}^{\prime}\right)$ by the Leray spectral sequence, and we see that $\pi^{*}$ preserves cohomology.

It now follows that the sections of $\pi^{*} \mathscr{F}$ are just the inverse image of the sections of $\mathscr{F}$, so the second assertion is immediate.

Many of our arguments use induction, based on the next two lemmas.

LEMMA 1.4. Let $\mathscr{E}=\left\{\mathscr{E}_{0}, \ldots, \mathscr{E}_{n}\right\}$ be an exceptional configuration on $V$. The $\mathscr{E}$-standard divisor classes are precisely the nonnegative sums of the classes $\mathscr{E}_{0}, \mathscr{E}_{0}-\mathscr{E}_{1}$, $2 \mathscr{E}_{0}-\mathscr{E}_{1}-\mathscr{E}_{2}$, and $-K_{i}, i>3$.

Proof. All nonnegative sums of the classes listed are clearly $\mathscr{E}$-standard. Conversely, suppose that $\mathscr{F}$ is $\mathscr{E}$-standard. If $\mathscr{F} \cdot \mathscr{E}_{i}=0$ for every $i$, then $\mathscr{F}$ is trivial; i.e., $\mathscr{F}$ is the zero sum. Otherwise, $\mathscr{F} \cdot \mathscr{E}_{i}>0$ for some $i$. Let $j$ be the largest index such that $\mathscr{F} \cdot \mathscr{E}_{j}>0$. If $0 \leqslant j \leqslant 2$, the result follows by a direct calculation, and if $j \geqslant 3$, by induction on $j$.

In particular, if $j=0$, then $\mathscr{F}=a \mathscr{E}_{0}, a>0$. If $j=1$, then $\mathscr{F}=a \mathscr{E}_{0}-b \mathscr{E}_{1}$ and $a \geqslant b>0$, so $\mathscr{F}=(a-b) \mathscr{E}_{0}+b\left(\mathscr{E}_{0}-\mathscr{E}_{1}\right)$. If $j=2$, then $\mathscr{F}=a \mathscr{E}_{0}-b \mathscr{E}_{1}-c \mathscr{E}_{2}$ and $a \geqslant b+c>b \geqslant c>0$ so

$$
\mathscr{F}=(a-b-c) \mathscr{E}_{0}+(b-c)\left(\mathscr{E}_{0}-\mathscr{E}_{1}\right)+c\left(2 \mathscr{E}_{0}-\mathscr{E}_{1}-\mathscr{E}_{2}\right)
$$

If $j \geqslant 3$, then, writing $\mathscr{F}$ as $a_{0} \mathscr{E}_{0}-a_{1} \mathscr{E}_{1}-\cdots-a_{j} \mathscr{E}_{j}$, we have $a_{0} \geqslant a_{1}+a_{2}+a_{3}$ $>a_{1} \geqslant a_{2} \geqslant \cdots \geqslant a_{j}>0$. A calculation shows that $\mathscr{F}+a_{j} K_{j}$ is $\mathscr{E}$-standard and that $\left(\mathscr{F}+a_{j} K_{j}\right) \cdot \mathscr{E}_{j}=0$. By induction on $j, \mathscr{F}+a_{j} K_{j}$ has the required form and thus so does $\mathscr{F}$.

LEMMA 1.5. Let $\mathscr{F}$ be an $\mathscr{E}$-standard class on $V$ for an exceptional cofiguration $\mathscr{E}=\left\{\mathscr{E}_{0}, \ldots, \mathscr{E}_{n}\right\}$ such that $\mathscr{F} \cdot \mathscr{E}_{i}=0, i \geqslant 8 ;$ i.e., $\mathscr{F}$ lies in Pic $V_{7}$. Then $\mathscr{F}$ is generated by global sections on $V$ and $h^{1}(V, \mathscr{F})=h^{2}(V, \mathscr{F})=0$.

Proof. Observe that the lemma is clearly true if $\mathscr{F}$ is trivial. Also, $\mathscr{E}_{0}, \mathscr{E}_{0}-\mathscr{E}_{1}$ and $2 \mathscr{E}_{0}-\mathscr{E}_{1}-\mathscr{E}_{2}$ are irreducible, having sections isomorphic to $\mathbf{P}^{1}$. Of course, $-K_{i}$ is irreducible on $V_{i}$ by hypothesis.

Let $\mathscr{F}$ be any of $\mathscr{E}_{0}, \mathscr{E}_{0}-\mathscr{E}_{1}, 2 \mathscr{E}_{0}-\mathscr{E}_{1}-\mathscr{E}_{2}$, or $-K_{1}, i \leqslant 7$. Let $j$ be the greatest index such that $\mathscr{F} \cdot \mathscr{E}_{j}>0$ and let $F$ be an irreducible, reduced section of $F$ on $V_{j}$.

Take $F, \mathscr{F}$ and $\mathscr{G}$ of $(0.4)$ to be $F, \mathscr{F}$ and $\mathscr{F}$, respectively. Since $\mathscr{F}-\mathscr{F}=\mathcal{O}_{V_{i}}$ and, by Lemma $1.3, h^{1}\left(V_{j}, \mathcal{O}_{V_{i}}\right)=h^{1}\left(\mathbf{P}^{2}, \mathcal{O}_{\mathbf{P}^{2}}\right)=0$, after an easy calculation $(0.4)$ says that $\mathscr{F}$ is generated by global sections on $F$. But $F$ is a section of $\mathscr{F}$ so $\mathscr{F}$ is generated by global sections off $F$ also.

Our first claim now follows from Lemmas 1.3 and 1.4, and the fact that a positive sum of classes generated by global sections is itself generated by global sections.

To prove the second claim, let $\mathscr{F}$ be $\mathscr{E}$-standard, i.e., $\mathscr{F}=a_{0} \mathscr{E}_{0}+a_{1}\left(\mathscr{E}_{0}-\mathscr{E}_{1}\right)+$ $a_{2}\left(2 \mathscr{E}_{0}-\mathscr{E}_{1}-\mathscr{E}_{2}\right)-\sum_{i=3}^{7} a_{i} K_{i}, a_{i} \geqslant 0$. We induct on $t=\sum a_{i}$. If $t=0$, then $\mathscr{F}=\mathcal{O}_{V}$ and we saw $h^{1}\left(V, \mathcal{O}_{V}\right)=0$, while $h^{2}\left(V, \mathcal{O}_{V}\right)=0$ by $(0.3)(\mathrm{b})$. Now assume $t>0$. 
Define $j$ to be the largest index such that $a_{j}>0$, and take $C$ to be an irreducible, reduced section on $V_{j}$ of the bundle whose coefficient is $a_{j}$. By induction and Lemma 1.3 we assume that $h^{2}\left(V, \mathscr{F}-\mathcal{O}_{V}(C)\right)=h^{1}\left(V, \mathscr{F}-\mathcal{O}_{V}(C)\right)=0$ and by $(0.4)$ and a calculation we see that $h^{1}\left(C, \mathcal{O}_{C} \otimes \mathscr{F}\right)=0$; obviously, $h^{2}\left(C, \mathcal{O}_{C} \otimes \mathscr{F}\right)=0$. Our claim, $h^{1}(V, \mathscr{F})=h^{2}(V, \mathscr{F})=0$, follows by taking cohomology of

$$
0 \rightarrow \mathscr{F}-\mathcal{O}_{V_{j}}(C) \rightarrow \mathscr{F} \rightarrow \mathscr{F} \otimes \mathcal{O}_{C} \rightarrow 0
$$

and using Lemma 1.3.

Proof of Theorem 1.1. (a) This is an immediate consequence of Lemma 1.4 and the fact that $-K$ is irreducible, and hence, for any exceptional configuration and any $i \geqslant 0,-K_{i}$ is effective.

(b) Suppose $\mathscr{F}$ is $\mathscr{E}$-excellent for an exceptional configuration $\mathscr{E}=\left\{\mathscr{E}_{0}, \ldots, \mathscr{E}_{n}\right\}$, and take $j$ to be the largest index such that $\mathscr{F} \cdot \mathscr{E}_{j}>0$. If $j \leqslant 7$, the conclusion is immediate from Lemma 1.5 and the theorem of Riemann-Roch for rational surfaces (0.2).

If $j>7$, we show in the next lemma that $\mathscr{F}+K_{j}$ is $\mathscr{E}$-excellent or trivial. By Lemma 1.3 , we may assume $j=n$ for the purpose of computing the cohomology of $\mathscr{F}$, in which case $-K_{j}=-K$ is irreducible. Taking the irreducible section $D$ of $-K$, we consider the sequence of bundles

$$
0 \rightarrow \mathscr{F}+K \rightarrow \mathscr{F} \rightarrow \mathscr{F} \otimes \mathcal{O}_{D} \rightarrow 0 .
$$

Since $\mathscr{F}$ is $\mathscr{E}$-standard, by Lemma 1.4 there are nonnegative integers $a_{0}, a_{1}, \ldots, a_{n}$ such that $\mathscr{F}=a_{0} \mathscr{E}_{0}+a_{1}\left(\mathscr{E}_{0}-\mathscr{E}_{1}\right)+a_{2}\left(2 \mathscr{E}_{0}-\mathscr{E}_{1}-\mathscr{E}_{2}\right)+a_{3}\left(-K_{3}\right)+\cdots+$ $a_{n}\left(-K_{n}\right)$ and, by assumption, $a_{n}>0$. By induction on $a_{8}+\cdots+a_{n}$, we assume

$$
h^{2}(V, \mathscr{F}+K)=h^{1}(V, \mathscr{F}+K)=0
$$

certainly $h^{2}\left(D, \mathscr{F} \otimes \mathcal{O}_{D}\right)=0$ and since $\operatorname{deg} \mathscr{F} \otimes \mathcal{O}_{D}=\mathscr{F} \cdot(-K)>0$ by hypothesis, $h^{1}\left(D, \mathscr{F} \otimes \mathcal{O}_{D}\right)=0$ by $(0.4)$.

Now taking cohomology we see $h^{2}(V, \mathscr{F})=h^{1}(V, \mathscr{F})=0$ and therefore $h^{0}(V, \mathscr{F})=\frac{1}{2}(\mathscr{F} \cdot \mathscr{F}-\mathscr{F} \cdot K)+1$ by $(0.2)$.

LEMMA 1.6. Let $\mathscr{F}$ be an $\mathscr{E}$-excellent class for an exceptional configuration $\mathscr{E}=$ $\left\{\mathscr{E}_{0}, \ldots, \mathscr{E}_{n}\right\}$, and suppose that $j \geqslant 8$ for $j$ the largest index such that $\mathscr{F} \cdot \mathscr{E}_{j}>0$. Then either $\mathscr{F}+K_{j}$ is $\mathscr{E}$-excellent or $\mathscr{F}=-K_{8}$ and $\mathscr{F}+K_{j}$ is trivial.

Proof. By Lemma 1.4, $\mathscr{F}+K_{j}$ must be $\mathscr{E}$-standard. For $j \geqslant 9, \mathscr{F}+K_{j}$ is $\mathscr{E}$-excellent since $\mathscr{F} \cdot K<0$ and $K_{j} \cdot K \leqslant 0$.

If $j=8$, it follows easily from Lemma 1.4 that $\mathscr{F} \cdot K<-1$ except when $\mathscr{F}=-K_{8}$. Thus $\mathscr{F}+K_{8}$ is trivial if $\mathscr{F}=-K_{8}$, and since $K \cdot K_{8}=1, \mathscr{F}+K_{8}$ is $\mathscr{E}$-excellent otherwise.

Proof of Proposition 1.2. If follows from Lemma 1.4 that if $\mathscr{F}$ is $\mathscr{E}$-standard and $\mathscr{F} \cdot K=0$ and if $\mathscr{F}$ lies in Pic $V_{9}$, then $\mathscr{F}$ is either trivial or a negative multiple of $K_{9}$.

Suppose $\mathscr{F}=-m K_{9}$ for $m \geqslant 0$. By Lemma 1.3 we may assume $V=V_{9}$. Consider the exact sequence

$$
0 \rightarrow(-m+1) K \rightarrow-m K \rightarrow \mathcal{O}_{D} \otimes(-m K) \rightarrow 0 .
$$


Since $\operatorname{deg} \mathcal{O}_{D} \otimes(-m K)=(-m K) \cdot(-K)=0$ and the arithmetic genus of $D$ is 1 , Riemann-Roch for curves [4, IV.1] says $h^{0}\left(D, \mathcal{O}_{D} \otimes(-m K)\right)=h^{1}\left(D, \mathcal{O}_{D} \otimes(-m K)\right)$, and these dimensions are 1 when $j^{*}(-m K)=0$, and zero otherwise. Taking cohomology, we see $h^{i}(V,(-m+1) K)=h^{i}(V,-m K), i=0,1$, as long as $l$ does not divide $m$, i.e., $h^{0}(V,-m K)=h^{0}(V,-l[m / l] K)$. Thus it is enough to verify that $h^{0}(V,-a l K)=a+1$ for $a \geqslant 0$.

The case that $a=0$ is trivial. So taking cohomology of the sequence above for $m=a l$ (keeping in mind that $h^{j}(V,-(a-1) l K)=h^{j}(V,-(a l-i) K), 1 \leqslant i \leqslant l$, $j=0,1)$, we see that $h^{0}(V,-a l K) \leqslant h^{0}(V,-(a-1) l K)+h^{0}\left(D, \mathcal{O}_{D}\right)$. By induction we may assume that the right side of this inequality equals $((a-1)+1)+$ $h^{0}\left(D, \mathcal{O}_{D}\right)$, or $a+1$. However, $h^{1}\left(V, \mathcal{O}_{V}\right)=0$, so the inequality is an equality for the case $a=1$. In particular, $I D$ moves in a pencil so $h^{0}(V,-a l K) \geqslant a+1$, and our result is proved for case (a).

We remark that if $j^{*}(K)$ has infinite order we have shown $h^{0}(V,-m K)=1$ for any $m \geqslant 0$, in agreement with the statement of the proposition, if we interpret $[\mathrm{m} / \mathrm{l}]$ as being zero.

Let $j$ be the largest index such that $\mathscr{F} \cdot \mathscr{E}_{j}>0$. If $j \leqslant 9$ we obtain the case treated above, so we may assume $j \geqslant 10$. By Lemma 1.3 , we may assume $j=n$ (i.e., $V_{j}=V$ ) for the purpose of computing cohomology. Thus $\mathscr{F}^{\prime}=\mathscr{F}+K$, so by Lemma $1.6 \mathscr{F}^{\prime}$ is $\mathscr{E}$-excellent, and $h^{1}\left(V, \mathscr{F}^{\prime}\right)=0$ by Theorem 1.1(b). Now take cohomology of the sequence

$$
0 \rightarrow \mathscr{F}^{\prime} \rightarrow \mathscr{F} \rightarrow \mathcal{O}_{D} \otimes \mathscr{F} \rightarrow 0 .
$$

If $j^{*}(\mathscr{F}) \neq 0$, then $h^{0}\left(D, \mathcal{O}_{D} \otimes \mathscr{F}\right)=0$, so we see $h^{0}\left(V, \mathscr{F}^{\prime}\right)=h^{0}(V, \mathscr{F})$. The desired result follows from Theorem 1.1(b) applied to $\mathscr{F}^{\prime}$. On the other hand, if $j^{*}(\mathscr{F})=0$, then $h^{0}\left(D, \mathcal{O}_{D} \otimes \mathscr{F}\right)=1$, so $h^{0}(V, \mathscr{F})=h^{0}\left(V, \mathscr{F}^{\prime}\right)+1$. Again, Theorem 1.1(b) applied to $\mathscr{F}^{\prime}$ gives the result.

2. The algorithm. The main result of this section is Theorem 2.1. Its proof is of particular interest because it gives an algorithm for determining whether an arbitrary class $\mathscr{F}$ on $V$ is effective, and if so, for subtracting off fixed components from $\mathscr{F}$ to obtain an almost excellent class $\mathscr{G}$. At the same time, our procedure produces an excepitonal configuration $\tilde{\mathscr{E}}$ for which $\mathscr{G}$ is almost $\tilde{\mathscr{E}}$-excellent. Our results of the previous section now suffice to determine $h^{0}(V, \mathscr{G})$, and hence also $h^{0}(V, \mathscr{F})$.

Our approach is similar to that of [5] wherein a procedure for determining the irreducible exceptional divisors is given. A modification of that procedure allows us to determine the dimension of the space of global sections for arbitrary divisors. We refer to the class of an exceptional divisor as an exceptional class and, as in [5], to an irreducible class $\mathscr{C}$ with $\mathscr{C}^{2}=-2$ and $\mathscr{C} \cdot K=0$ as a nodal class.

THEOREM 2.1. Let $\mathscr{F}$ be a divisor class on $V$. There exists a class $\mathscr{G}$ and an exceptional configuration $\tilde{\mathscr{E}}=\left\{\tilde{\mathscr{E}}_{0}, \ldots, \tilde{\mathscr{E}}_{n}\right\}$ on $V$ such that $\mathscr{F}-\mathscr{G}$ is effective, in particular being a nonnegative sum of nodal and exceptional classes and $-K$ when $\mathscr{F}$ is effective; $h^{0}(V, \mathscr{G})=h^{0}(V, \mathscr{F})$; and one of the following holds:

(a) $\mathscr{G} \cdot \tilde{\mathscr{E}}_{0}<0$ and hence $\mathscr{G}$ is not effective, or 
(b) $\mathscr{G}$ is almost $\tilde{\mathscr{E}}$-excellent, and hence $\mathscr{G}$ is effective and $h^{0}(V, \mathscr{G})$ is given by Theorem 1.1 or Proposition 1.2.

Lemma 2.2, due to Looijenga [5, I.4.1 and I.5.2] in somewhat modified form, is central to the theorem's proof.

Given an exceptional configuration $\mathscr{E}=\left\{\mathscr{E}_{0}, \ldots, \mathscr{E}_{n}\right\}$ we have the roots $r_{i} \in$ Pic $V$, $i=0, \ldots, n-1$, defined in $\S 0$. To each is associated the reflection $s_{i}$ : Pic $V \rightarrow \operatorname{Pic} V$ defined by $s_{i}(x)=x+\left(x \cdot r_{i}\right) r_{i}$. (The significance of these operations seems first to have been realized by DuVal [2] (see also [6]). They occur in [5] in related but more general circumstances and are implicit in [7].)

Lemma 2.2. (1) The collection $\mathscr{E}^{\prime}=\left\{\mathscr{E}_{0}^{\prime}=s_{i}\left(\mathscr{E}_{0}\right), \ldots, \mathscr{E}_{n}^{\prime}=s_{i}\left(\mathscr{E}_{n}\right)\right\}$ of classes is an exceptional configuration iff $r_{i}$ is not effective.

(2) The class $r_{i}$ is effective iff it is irreducible iff $j^{*}\left(r_{i}\right)=0$ (for $j^{*}: \operatorname{Pic} V \rightarrow \operatorname{Pic} D$ defined earlier).

Proof. (1) Recall that, as in (0.1), $\mathscr{E}$ represents $V$ as a blowing-up of $\mathbf{P}^{2}$ at the points, possibly infinitely near, $p_{1}, p_{2}, \ldots, p_{n}$.

First suppose $i \neq 0$. Then $s_{i}$ just transposes $\mathscr{E}_{i}$ and $\mathscr{E}_{i+1}$, which gives an exceptional configuration precisely when $p_{i+1}$ is not infinitely near $p_{i}$, i.e., when $\mathscr{E}_{i}-\mathscr{E}_{i+1}=r_{i}$ is not effective.

Now suppose $i=0$. The operation $s_{0}$ is an element of order 2 in GL(Pic $V$ ) and preserves the intersection form. In the basis $\mathscr{E}^{\prime}, r_{0}$ has the expression $-\mathscr{E}_{0}^{\prime}+\mathscr{E}_{1}^{\prime}+\mathscr{E}_{2}^{\prime}$ $+\mathscr{E}_{3}^{\prime}$. By $(0.3)\left(\right.$ a), $r_{0}$ cannot be effective if $\mathscr{E}^{\prime}$ is an exceptional configuration, since $r_{0} \cdot \mathscr{E}_{0}^{\prime}<0$.

Conversely, suppose $\mathscr{E}^{\prime}$ is not an exceptional configuration. Then we must show that $r_{0}$ is effective. By Lemma 1.3 it is enough to check this on $V_{3}$, i.e., we may assume that $V$ is a blowing-up of $\mathbf{P}^{2}$ at the three points $p_{1}, p_{2}$ and $p_{3}$ which give rise to $\mathscr{E}_{1}, \mathscr{E}_{2}$, and $\mathscr{E}_{3}$. The points $p_{2}$ and $p_{3}$ may, of course, be infinitely near points of $\mathbf{P}^{2}$.

It is more convenient to prove the contrapositive, so we assume $r_{0}$ is not effective. Since $r_{0}=\mathscr{E}_{0}-\mathscr{E}_{1}-\mathscr{E}_{2}-\mathscr{E}_{3}$, this means precisely that the points are not collinear, i.e., $p_{1}, p_{2}, p_{3}$ are not simultaneously points (or infinitely near points) of any line $L$ in $\mathbf{P}^{2}$. After possibly permuting the points, permissible by the first part of this proof, we have three cases: the three points $p_{1}, p_{2}$, and $p_{3}$ are not collinear and either

(i) $p_{1}, p_{2}$, and $p_{3}$ are distinct points of $\mathbf{P}^{2}$, or

(ii) $p_{1}$ and $p_{3}$ are distinct points of $\mathbf{P}^{2}$, and $p_{2}$ is infinitely near $p_{1}$, or

(iii) $p_{3}$ and $p_{2}$ are both infinitely near $p_{1}$.

We will only do case (iii); the others are similar and easier. Now $p_{2}$ is a point of the exceptional curve $E_{1}$ on $V_{1}$ resulting from blowing up $p_{1}$. The total transform of $E_{1}$ on the blowing-up $V_{2}$ of $p_{2}$ is $N+E_{2}$, where $N$ is the proper transform of $E_{1}$. The class of $N$ in Pic $V_{2}$ is just $r_{1}$.

Now $p_{3}$ lies on $N+E_{2}$, but in fact $p_{3}$ cannot lie on $N$. This would imply that $r_{1}-\mathscr{E}_{3} \in \mathrm{Pic} V_{3}$ is irreducible, and $-K \cdot\left(r_{1}-\mathscr{E}_{3}\right)=-1$ would contradict our having $-K$ irreducible. Thus $p_{3}$ lies on $E_{2} \backslash N$, and since the three points are not collinear, $p_{3}$ avoids the point where the proper transform $L^{*}$ of the line $L$ through $p_{1}$ and $p_{2}$ 
meets $E_{2}$. If we call by $N_{2}$ the proper transform of $E_{2}$ on $V_{3}$, we have the following schematic diagram of the blowings-up $V_{3} \rightarrow V_{2} \rightarrow V_{1} \rightarrow \mathbf{P}^{2}$ :

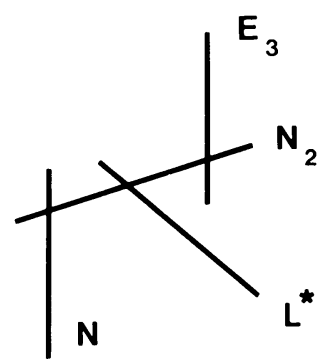

Therefore, $\mathscr{E}_{3}^{\prime}$, being just $\mathscr{E}_{0}-\mathscr{E}_{1}-\mathscr{E}_{2}$, is the class of $L^{*} ; \mathscr{E}_{2}^{\prime}=\mathscr{E}_{0}-\mathscr{E}_{1}-\mathscr{E}_{3}$ that of $L^{*}+N_{2}$; and $\mathscr{E}_{1}^{\prime}=\mathscr{E}_{0}-\mathscr{E}_{2}-\mathscr{E}_{3}$ that of $L^{*}+N_{2}+N$. By Castelnuovo's criterion (see [4, p. 414]) we can contract $\mathscr{E}_{3}^{\prime}, \mathscr{E}_{2}^{\prime}$, and $\mathscr{E}_{1}^{\prime}$ in order, obtaining a smooth rational surface $V^{\prime}$ with Pic $V^{\prime}$ having rank 1, i.e., $V^{\prime}$ is isomorphic to $\mathbf{P}^{2}$.

Thus $\mathscr{E}^{\prime}=\left\{\mathscr{E}_{0}^{\prime}, \mathscr{E}_{1}^{\prime}, \mathscr{E}_{2}^{\prime}, \mathscr{E}_{3}^{\prime}\right\}$ is an exceptional configuration if $\mathscr{E}_{0}^{\prime}=2 \mathscr{E}_{0}-\mathscr{E}_{1}-\mathscr{E}_{2}$ $-\mathscr{E}_{3}$ is the class $\mathscr{L}$ of a line in $V^{\prime}$. Now $\mathscr{L}$ is uniquely determined by the conditions that $\mathscr{L} \cdot \mathscr{E}_{i}^{\prime}=0, i=1,2,3$, that $\mathscr{L}^{2}=1$, and that $\mathscr{L}$ meets $\mathscr{E}_{0}$ positively, i.e., $\mathscr{L} \cdot \mathscr{E}_{0}>0$. The only class satisfying these conditions is $\mathscr{E}_{0}^{\prime}$.

(2) Consider the sequence of morphisms (0.1) associated to the exceptional configuration $\mathscr{E}$. For $i \geqslant 1, r_{i}$ is effective iff $p_{i+1}$ is a point of $E_{i}$, in which case $r_{i}$ is irreducible on $V_{i+1}$. But the points $p_{1}, \ldots, p_{n}$ are chosen to be points, possibly infinitely near, of a reduced, irreducible cubic in $\mathbf{P}^{2}$.

Refer to this cubic and its proper transform on the blowing-up $V_{j}, j \geqslant 0$, as $D$. We have on $V_{i+1}$ that

$$
D \cdot r_{i}=-K_{i+1} \cdot r_{i}=\left(3 \mathscr{E}_{0}-\mathscr{E}_{1}-\cdots-\mathscr{E}_{i+1}\right) \cdot\left(\mathscr{E}_{i}-\mathscr{E}_{i+1}\right)=0 .
$$

Thus if $r_{i}$ is irreducible, it does not meet $D$, and so the points $p_{i+2}, \ldots, p_{n}$, being points of $D$, cannot lie on $r_{i}$, i.e., $r_{i}$ is irreducible on $V$. Moreover, $r_{i}$ is effective iff $E_{i}$ and $E_{i+1}$ meet $D$ in the same point; that is, iff $j^{*}\left(r_{i}\right)=0$.

One can reason in a similar way for $r_{0}$.

Our proof of Theorem 2.1 is algorithmic, or inductive, if you like. To control the induction, for each exceptional configuration $\mathscr{E}=\left\{\mathscr{E}_{0}, \ldots, \mathscr{E}_{n}\right\}$, we will associate to each class $\mathscr{F}$ on $V$ three integers: the $\mathscr{E}$-shuffle number, the $\mathscr{E}$-positivity, and the $\mathscr{E}$-degree.

Suppose $\mathscr{F}=a_{0} \mathscr{E}_{0}+a_{1} \mathscr{E}_{1}+\cdots+a_{n} \mathscr{E}_{n}$ in terms of $\mathscr{E}$. The $\mathscr{E}$-shuffle number of $\mathscr{F}$ is $\sum_{1 \leqslant i<j \leqslant n}\left(a_{i}-a_{j}\right) \nu\left(a_{i}-a_{j}\right)$, where $\nu: \mathbf{Z} \rightarrow \mathbf{Z}$ is defined to be zero for nonpositive integers and one otherwise. The $\mathscr{E}$-positivity is $\sum_{i \geqslant 1} \nu\left(a_{i}\right)$ and the $\mathscr{E}$-degree is $a_{0}$.

We observe that the shuffle number is never negative and is zero precisely when $a_{1} \leqslant a_{2} \leqslant \cdots \leqslant a_{n}$. In particular, $\mathscr{F}$ is $\mathscr{E}$-standard precisely when the $\mathscr{E}$-shuffle number and $\mathscr{E}$-positivity are zero and $a_{0}+a_{1}+a_{2}+a_{3} \geqslant 0$.

We now define three operations that, given a class $\mathscr{F}$ and a configuration $\mathscr{E}=\left\{\mathscr{E}_{0}, \ldots, \mathscr{E}_{n}\right\}$, produce a class $\mathscr{F}^{\prime}$ and a configuration $\mathscr{E}^{\prime}$ such that $\mathscr{F}-\mathscr{F}^{\prime}$ is effective; $h^{0}(V, \mathscr{F})=h^{0}(V, \mathscr{F})$; and one number among the shuffle number, 
positivity, and degree decreases, with the degree never increasing:

(A) Let $r_{i}=\mathscr{E}_{i}-\mathscr{E}_{i+1}, i=1, \ldots, n-1$, and suppose $r_{i} \cdot \mathscr{F}<0$. If $j^{*}\left(r_{i}\right)=0$, i.e., if $r_{i}$ is irreducible (Lemma 2.2(2)), take $\mathscr{F}^{\prime}=\mathscr{F}-r_{i}$ and $\mathscr{E}^{\prime}=\mathscr{E}$. If $j^{*}\left(r_{i}\right) \neq 0$, i.e., if $r_{i}$ is not effective (Lemma $\left.2.2(2)\right)$, take $\mathscr{F}^{\prime}=\mathscr{F}$ and $\mathscr{E}^{\prime}=\left\{s_{i}\left(\mathscr{E}_{0}\right), \ldots, s_{i}\left(\mathscr{E}_{n}\right)\right\}$.

Clearly $\mathscr{F}-\mathscr{F}^{\prime}$ is nodal or trivial. We show $h^{0}(V, \mathscr{F})=h^{0}\left(V, \mathscr{F}^{\prime}\right)$. This is immediate except in the case that $r_{i}$ is irreducible. But if $\mathscr{F}$ is effective, then $r_{i}$ is a fixed component of $\mathscr{F}$, whence $h^{0}(V, \mathscr{F})=h^{0}\left(V, \mathscr{F}^{\prime}\right)$. If $\mathscr{F}^{\prime}$ is effective, then so is $\mathscr{F}$, since $\mathscr{F}^{\prime}+r_{i}=\mathscr{F}$. Therefore $h^{0}(V, \mathscr{F})=0$ exactly when $h^{0}\left(V, \mathscr{F}^{\prime}\right)=0$.

Also $\mathscr{E}^{\prime}$ is an exceptional configuration. In the first case it is obvious and in the second it follows from Lemma 2.2(1).

We remark that the $\mathscr{E}$-degree of $\mathscr{F}$ equals the $\mathscr{E}^{\prime}$-degree of $\mathscr{F}^{\prime}$, and the $\mathscr{E}$-shuffle number of $\mathscr{F}$ is greater than the $\mathscr{E}^{\prime}$-shuffle number of $\mathscr{F}^{\prime}$.

(B) Suppose $\mathscr{F}$ is a class for which the $\mathscr{E}$-positivity is nonzero and such that $\mathscr{F} \cdot r_{i} \geqslant 0$ for all $i \geqslant 1$, i.e., the $\mathscr{E}$-shuffle number of $\mathscr{F}$ is zero. Then we take $\mathscr{E}^{\prime}=$ $\mathscr{E}$ and, if we write $\mathscr{F}=a_{0} \mathscr{E}_{0}+\cdots+a_{n} \mathscr{E}_{n}$, we take $\mathscr{F}^{\prime}=a_{0} \mathscr{E}_{0}+\sum a_{i} \mathscr{E}_{i}$ for $a_{i}<0$, $i>0$. Reasoning similar to that used in (A) shows that $\mathscr{F}-\mathscr{F}^{\prime}$ is effective, indeed, it is a sum of exceptional classes, and $h^{0}(V, \mathscr{F})=h^{0}\left(V, \mathscr{F}^{\prime}\right)$. Also, the positivity becomes zero and the degree is unchanged.

(C) First assume that Pic $V$ has rank $\geqslant 4$. Let $r_{0}=\mathscr{E}_{0}-\mathscr{E}_{1}-\mathscr{E}_{2}-\mathscr{E}_{3}$ and suppose that $r_{0} \cdot \mathscr{F}<0$. Then we take $\mathscr{F}^{\prime}=\mathscr{F}-r_{0}$ and $\mathscr{E}^{\prime}=\mathscr{E}$ if $j^{*}\left(r_{0}\right)=0$; and $\mathscr{F}^{\prime}=$ $\mathscr{F}$ and $\mathscr{E}^{\prime}=s_{0}(\mathscr{E})$ if $j^{*}\left(r_{0}\right) \neq 0$. As in (A), $\mathscr{E}^{\prime}$ is an exceptional configuration, $\mathscr{F}-\mathscr{F}^{\prime}$ is nodal or trivial and $h^{0}(V, \mathscr{F})=h^{0}\left(V, \mathscr{F}^{\prime}\right)$. Moreover, this operation reduces the degree, although the positivity and shuffle number may increase.

Note 2.3. If Pic $V$ has rank 1, we do not define (C). If the rank is 2 or 3 , replace $r_{0}$ by $r_{-2}$ or $r_{-1}$ (see $\S 0$ ), respectively, and define (C) as in the case $j^{*}\left(r_{0}\right)=0$. We note that $\mathscr{F}-\mathscr{F}^{\prime}$ is an exceptional class in the case that the rank is 3 . When the rank is 2 , $\mathscr{F}-\mathscr{F}^{\prime}$, though effective, is neither exceptional nor nodal. But since $r_{-2}$ is an irreducible class with nonnegative self-intersection, $\mathscr{F}$ is not effective if $\mathscr{F} \cdot r_{-2}<0$.

Proof OF THEOREM 2.1. We begin with a class $\mathscr{F}$ and an exceptional configuration $\mathscr{E}$. If the $\mathscr{E}$-degree of $\mathscr{F}$ is negative we are done, taking the class $\mathscr{G}$ in the statement of the theorem to be $\mathscr{F}$. Otherwise, repeatedly applying the operation (A) (if necessary) we eventually obtain from $\mathscr{F}$ and $\mathscr{E}$ a class $\mathscr{F}^{\prime}$ and a configuration $\mathscr{E}$ ' such that the $\mathscr{E}^{\prime}$-shuffle number of $\mathscr{F}^{\prime}$ is zero, $\mathscr{F}-\mathscr{F}^{\prime}$ is trivial or a sum of nodal classes and $h^{0}(V, \mathscr{F})=h^{0}\left(V, \mathscr{F}^{\prime}\right)$.

After applying (B), if necessary, we can assume that the $\mathscr{E}^{\prime}$-positivity of $\mathscr{F}^{\prime}$ is zero; now $\mathscr{F}-\mathscr{F}^{\prime}$ may also include exceptional classes. If the $\mathscr{E}^{\prime}$-degree of $\mathscr{F}^{\prime}$ is negative, we are done, as before. If not, consider $\mathscr{F}^{\prime} \cdot t$, where $t=r_{-2}^{\prime}, r_{-1}^{\prime}$, or $r_{0}^{\prime}$, depending on whether rk Pic $V$ is 2,3 , or 4 or more, respectively. If $\mathscr{F}^{\prime} \cdot t \geqslant 0$, then $\mathscr{F}^{\prime}$ is $\mathscr{E}^{\prime}$-standard. If $\mathscr{F}^{\prime} \cdot t<0$, apply (C) (viz. Note 2.3) and denote the result by $\mathscr{F}^{\prime \prime}$ and $\mathscr{E}^{\prime \prime}$, and note that the $\mathscr{E}^{\prime \prime}$-degree of $\mathscr{F}^{\prime \prime}$ is less than the $\mathscr{E}$-degree of $\mathscr{F}$.

By induction on the degree it is clear that by repeating the operations of the last two paragraphs we obtain a class $\mathscr{G}^{*}$ and a configuration $\mathscr{E}^{*}$ such that $\mathscr{F}-\mathscr{G}^{*}$ is effective, $h^{0}(V, \mathscr{F})=h^{0}\left(V, \mathscr{G}^{*}\right)$ and either the $\mathscr{E}^{*}$-degree of $\mathscr{G}^{*}$ is negative, or $\mathscr{G}^{*}$ is $\mathscr{E}^{*}$-standard. Moreover, if $\mathscr{G}^{*}$ is $\mathscr{E}^{*}$-standard, then, by Theorem 1.1(a), $\mathscr{F}$ is 
effective and our procedure results in having $\mathscr{F}-\mathscr{G} *$ equal to a nonnegative sum of nodal and exceptional classes.

In the case that $\mathscr{G}^{*}$ is $\mathscr{E}^{*}$-standard, if $K \cdot \mathscr{G}^{*} \leqslant 0$, then $\mathscr{G}^{*}$ is almost $\mathscr{E}^{*}$-excellent; so taking $\mathscr{G}=\mathscr{G}^{*}$ and $\tilde{\mathscr{E}}=\mathscr{E}^{*}$, we are done. If not, it follows from Lemma 1.4 that $j \geqslant 10$, where $j$ is the largest index with $\mathscr{G} \cdot \mathscr{E}_{j}^{*}>0$. Now $\mathscr{G}^{*}$ is effective (Theorem $1.1(\mathrm{a})),-K_{j}$ is a fixed component of $\mathscr{G}^{*}$ and $\left(\mathscr{G}^{*}+K_{j}\right) \cdot K<\mathscr{G}^{*} \cdot K$. However, $\mathscr{G}^{*}+K_{j}$ is still $\mathscr{E}^{*}$-standard (cf. Lemma 1.4 ), so repeating this process and inducting on $\mathscr{G}^{*} \cdot K$, we obtain an $\mathscr{E}^{*}$-standard class $\mathscr{G}$ with $K \cdot \mathscr{G} \leqslant 0$. Thus $\mathscr{G}$ is almost $\mathscr{E}^{*}$-excellent and since $-K_{j}$ is a sum of $-K$ and exceptional classes, $\mathscr{F}-\mathscr{G}$ is a nonnegative sum of nodal and exceptional classes and $-K$. Taking $\tilde{\mathscr{E}}=\mathscr{E}^{*}$ we are done.

We will use the following corollary in the next section.

COROllary 2.4. Let $\mathscr{F} \in$ Pic $V$ and suppose $\mathscr{F} \cdot \mathscr{C} \geqslant 0$ for any irreducible class $\mathscr{C}$. Then $\mathscr{F}$ is almost excellent.

Proof. If $\mathscr{F} \cdot \mathscr{C} \geqslant 0$ for any irreducible class $\mathscr{C}$, the procedure of the proof of Theorem 2.1 leaves $\mathscr{F}$ unchanged but produces a configuration $\tilde{\mathscr{E}}$ for which either $\mathscr{F} \cdot \tilde{\mathscr{E}}_{0}<0$ or $\mathscr{F}$ is almost $\tilde{\mathscr{E}}$-excellent. But $\tilde{\mathscr{E}}_{0}$ is irreducible so $\mathscr{F} \cdot \tilde{\mathscr{E}}_{0} \geqslant 0$ and $\mathscr{F}$ must be almost $\tilde{\mathscr{E}}$-excellent.

3. Fixed components and base points. In this section we determine (Theorem 3.1) the fixed components of any effective class, and (Corollary 3.4) the base points for any effective class without fixed components.

Actually, it is enough to determine the fixed components of almost excellent classes. By Corollary 3.2 a class $\mathscr{F}$ is almost excellent iff $\mathscr{F} \cdot \mathscr{C} \geqslant 0$ for every irreducible class $\mathscr{C}$. Therefore, if $\mathscr{F}$ is effective but not almost excellent, there is an irreducible $\mathscr{C}$ with $\mathscr{F} \cdot \mathscr{C}<0$ and $\mathscr{C}$ is a fixed component of $\mathscr{F}$. Our proof of Theorem 2.1, which reduces $\mathscr{F}$ to being almost excellent, gives a procedure for finding all such $\mathscr{C}$ (and in particular shows that $\mathscr{C}$ must be $-K$, or, for some configuration $\mathscr{E}=\left\{\mathscr{E}_{0}, \ldots, \mathscr{E}_{n}\right\}, \mathscr{E}_{i}$ or $r_{i}$ ).

THEOREM 3.1. Let $\mathscr{F}$ be an almost $\mathscr{E}$-excellent class for some exceptional configuration $\mathscr{E}=\left\{\mathscr{E}_{0}, \ldots, \mathscr{E}_{n}\right\}$. Then $\mathscr{F}$ has a fixed component iff one of the conditions below holds:

(a) $\mathscr{F} \cdot K=0$ but $j^{*}(\mathscr{F}) \neq 0$; here $-K$ is a fixed component; or

(b) $\mathscr{F}=-(m+2) K_{9}+\left(\mathscr{E}_{9}-\mathscr{E}_{10}\right), m \geqslant 0, j^{*}\left(-K_{9}\right)=0$, and $j^{*}\left(\mathscr{E}_{9}-\mathscr{E}_{10}\right)=0$; here $\mathscr{E}_{9}-\mathscr{E}_{10}$ is a fixed component; or

(c) for some $i, \mathscr{F} \cdot \mathscr{E}_{i}=0$ and $j^{*}\left(\mathscr{F}-\mathscr{E}_{i}\right)=0$; here $\mathscr{E}_{i}$ is a fixed component; or

(d) $\mathscr{F}=-(m+1) K_{9}+\mathscr{E}_{9}, m \geqslant 0$, and $j^{*}\left(-K_{9}\right)=0$; here $\mathscr{E}_{9}$ is a fixed component of $\mathscr{F}$.

Our proof follows Lemma 3.3.

We can now characterize the almost excellent classes:

COROllaRy 3.2. A class $\mathscr{F}$ is almost excellent iff $\mathscr{F} \cdot \mathscr{C} \geq 0$ for every irreducible class $\mathscr{C}$ 
Proof. By Corollary 2.4 , if $\mathscr{F} \cdot \mathscr{C} \geqslant 0$ for every irreducible $\mathscr{C}$, then $\mathscr{F}$ is almost excellent. Conversely, it will follow that $\mathscr{F} \cdot \mathscr{C} \geqslant 0$ for every irreducible class if we show that an almost excellent class $\mathscr{F}$ meets its fixed components nonnegatively.

If $\mathscr{F}$ has no fixed components we are done. Otherwise we are reduced to one of the four cases of Theorem 3.1 .

In cases (b) and (d), it is clear that $\mathscr{F}$ meets its fixed components nonnegatively.

In case (c), let $j$ be the least index with $\mathscr{F} \cdot \mathscr{E}_{j}=0$. Then $\mathscr{F}$ lies in Pic $V_{j-1}$ (cf. $(0.1)$ ), and as a divisor class on $V_{j-1}, \mathscr{F}$ satisfies at most the conditions of case (d). Thus any fixed component of $\mathscr{F}$ on $V$ must be a component of some $\mathscr{E}_{i}$, and so of the form $\mathscr{E}_{r}$ or $\mathscr{E}_{r}-\mathscr{E}_{s}, 0<r<s$. By almost $\mathscr{E}$-excellence, these meet $\mathscr{F}$ nonnegatively.

In case (a), define $j$ as above. Then $-K_{j-1}$ is a fixed component of $\mathscr{F}$. It follows from Lemma 1.4 that either $\mathscr{F}+K_{j-1}$ is a case previously treated or it is a nonnegative multiple of $-K_{9}$, so it meets its fixed components nonnegatively. Since $-K_{j-1}$ also meets all irreducible classes but $-K$ nonnegatively, and since $\mathscr{F} \cdot(-K)=0$, we conclude $\mathscr{F}$ meets its fixed components nonnegatively.

The following result is used in the proof of Theorem 3.1.

LEMMA 3.3. Let $\mathscr{F}$ be $\mathscr{E}$-excellent for an exceptional configuration $\mathscr{E}=\left\{\mathscr{E}_{0}, \ldots, \mathscr{E}_{n}\right\}$.

(1) If $\mathscr{F}$ lies in Pic $V_{i}$ for some $i=0, \ldots, n$, then $-K_{i}$ is not a fixed component of $\mathscr{F}$ on $V_{i}$.

(2) If $\mathscr{F} \cdot K \leqslant-2$, then $\mathscr{F}$ is generated by global sections.

Proof. If $i \leqslant 7$, then $\mathscr{F}$ is generated by global sections by Lemma 1.5 so both (1) and (2) follow for this case.

Since for $j \geqslant i,-K_{j}$ is a fixed component of $\mathscr{F}$ precisely when $-K_{i}$ is, we need only prove (1) under the conditions that $\mathscr{F}$ lie in Pic $V_{i}$, but not in Pic $V_{i-1}$.

If $i \geqslant 8$, then either $\mathscr{F}+K_{i}$ is trivial or $\mathscr{E}$-excellent by Lemma 1.6. In particular, $h^{0}\left(V_{i}, \mathscr{F}+K_{i}\right)$ is positive and we obtain the following exact sequence by taking cohomology of the appropriate sequence of sheaves and using $h^{1}\left(V_{i}, \mathscr{F}+K_{i}\right)=0$ (Theorem 1.1(b)):

$$
0 \rightarrow H^{0}\left(V_{i}, \mathscr{F}+K_{i}\right) \rightarrow H^{0}\left(V_{i}, \mathscr{F}\right) \rightarrow H^{0}\left(D, \mathscr{F} \otimes \mathcal{O}_{D}\right) \rightarrow 0 .
$$

Now $h^{0}\left(D, \mathscr{F} \otimes \mathcal{O}_{D}\right) \geqslant 1$ since $\operatorname{deg}\left(\mathscr{F} \otimes \mathcal{O}_{D}\right) \geqslant 1$ and $D$ has genus 1 , so we see $\mathscr{F}+K_{i}$ has fewer sections than $\mathscr{F}$. That is, $-K_{i}$ is not a fixed component of $\mathscr{F}$. This proves (1).

To prove (2), note that, by $(0.4), \mathscr{F} \cdot K \leqslant-2$ means that, on $V_{i}, \mathscr{F}$ is generated by global sections along $D$.

We finish the proof by induction. By Lemma $1.6, \mathscr{F}^{\prime}=\mathscr{F}+\left(\mathscr{E}_{i} \cdot \mathscr{F}\right) K_{i}$ is trivial or $\mathscr{E}$-excellent. In the former case, $\mathscr{F}^{\prime}$ is generated by global sections on $V_{i}$ and hence so is $\mathscr{F}$. By Lemma 1.3 , the same is true on $V$. In the latter case, it follows from Lemma 1.4 that $\mathscr{F}^{\prime} \cdot K \leqslant-2$, so, by induction on $i, \mathscr{F}^{\prime}$ is generated by global sections on $V_{i-1}$. As before, we conclude $\mathscr{F}$ is generated by global sections on $V$.

Proof of Theorem 3.1. We first show that the conditions given are sufficient. For case (a) it follows from Proposition 1.2. For case (b), $\mathscr{E}_{9}-\mathscr{E}_{10}$ is irreducible by 
Lemma 2.2(2). A calculation using Proposition 1.2(a), (b) shows

$$
h^{0}\left(V, \mathscr{F}-\left(\mathscr{E}_{9}-\mathscr{E}_{10}\right)\right)=h^{0}(V, \mathscr{F}),
$$

so $\mathscr{E}_{9}-\mathscr{E}_{10}$ is a fixed component of $\mathscr{F}$. Case (d) follows, likewise by a calculation using Theorem 1.1(b) and Proposition 1.2(a).

Consider case (c). Let $j$ be the largest index with $\mathscr{F} \cdot \mathscr{E}_{j}>0$, i.e. $\mathscr{F}$ lies in Pic $V_{j}$ but not in Pic $V_{j-1}$. Denoting the anticanonical divisor on $V_{j}$ by $D$, Lemma 3.3(1) implies $D$ is not a fixed component of $\mathscr{F}$. Therefore $j^{*}\left(\mathscr{F}-\mathscr{E}_{i}\right)=0$ means that $\mathscr{F}$ meets $D$ at one point $p$ which is a base point for $\mathscr{F}$ on $V_{j}$ and that $\mathscr{E}_{i}$ is the blowing-up of a point infinitely near to $p$. In particular, $\mathscr{E}_{i}$ is a fixed component of $\mathscr{F}$ on $V$.

For the converse, we first consider the case that $\mathscr{F}$ is $\mathscr{E}$-excellent, i.e., that $\mathscr{F} \cdot K<0$. If $\mathscr{F} \cdot K \leqslant-2$, then $\mathscr{F}$ is generated by global sections by Lemma 3.3(2), and hence has neither fixed components nor base points.

Suppose now that $\mathscr{F} \cdot K=-1$. Assuming case (c) of the theorem does not hold, if $\mathscr{F} \cdot \mathscr{E}_{i}=0$, then $j^{*}\left(\mathscr{F}-\mathscr{E}_{i}\right) \neq 0$. Since $-K$ is not a fixed component of $\mathscr{F}$ (Lemma 3.3(1)), $\mathscr{F}$ and $\mathscr{E}_{i}$ meet $D$ at different points, so $\mathscr{E}_{i}$ cannot be a fixed component of $\mathscr{F}$. But then $\mathscr{F} \cdot \mathscr{E}_{i}=0$ means that $\mathscr{F}$ and $\mathscr{E}_{i}$ do not meet at all, i.e., $\mathscr{F}$ is generated by global sections along the unique section of $\mathscr{E}_{i}$. Now by Lemma 1.3 we may reduce to the case that $\mathscr{F} \cdot \mathscr{E}_{i}>0$ for all $i=0, \ldots, n$.

If $n \leqslant 7$, then $\mathscr{F}$ is generated by global sections (Lemma 1.5). If $n=8$, then Lemma 1.4 and $\mathscr{F} \cdot K=-1$ imply that $\mathscr{F}=-K$. But $-K$ is irreducible and not fixed so $\mathscr{F}$ has no fixed components.

If $n=9$, it follows from Lemma 1.4 that $\mathscr{F}$ is $-K_{8}-m K$ for some $m \geqslant 1$. We can rewrite this in the form $-(m+1) K+\mathscr{E}_{9}$. By Lemma $3.3(1),-K$ is not a fixed component of $\mathscr{F}$, so if $\mathscr{F}$ has a fixed component, it must be $\mathscr{E}_{9}$, which therefore must be a fixed component of $-K_{8}$. This would imply that

$$
2=h^{0}\left(V,-K_{8}\right)=h^{0}\left(V,-K_{8}-\mathscr{E}_{9}\right) .
$$

It follows by Proposition 1.2 that $j^{*}\left(-K_{8}-\mathscr{E}_{9}\right)=0$, but since $-K_{8}-\mathscr{E}_{9}=-K$, case (d) not holding says that this cannot happen.

Finally, if $n \geqslant 10$, then $\mathscr{F}+K$ is $\mathscr{E}$-excellent (Lemma 1.6) and $(\mathscr{F}+K) \cdot K \leqslant-2$. By Lemma 3.3(2), $\mathscr{F}+K$ is generated by global sections. But by Lemma 3.3(1), $-K$ is not a fixed component of $\mathscr{F}$ so $\mathscr{F}$ has no fixed components.

Now suppose $\mathscr{F}$ is almost $\mathscr{E}$-excellent and $\mathscr{F} \cdot K=0$. We will show that cases (a) and (b) not holding implies that $\mathscr{F}$ is generated by global sections.

By Lemma 1.3 , it is enough to prove that $\mathscr{F}$ is generated by global sections in the case that $\mathscr{F} \cdot \mathscr{E}_{i}>0, i=1, \ldots, n$.

First, case (a) not holding implies that $j^{*}(\mathscr{F})=0$, and a calculation using Proposition 1.2 shows that $-K$ is not a fixed component of $\mathscr{F}$. Now consider some cases.

If $n \geqslant 11$, then (cf. Lemma 1.4$) \mathscr{F}+K$ is $\mathscr{E}$-excellent; indeed, $(\mathscr{F}+K) \cdot K \leqslant-2$, so by Lemma $3.3 \mathscr{F}+K$ is generated by global sections, and hence so is $\mathscr{F}$ off $D$. But $-K$ is not a fixed component of $\mathscr{F}$ and $\mathscr{F} \cdot(-K)=0$, so $\mathscr{F}$ is generated by global sections also at any point of $D$. 
If $n \geqslant 9$, then $n=9$ and $\mathscr{F}=-m K, m \geqslant 0$ (cf. Lemma 1.4). But any base point of $\mathscr{F}$ would be a base point of $-K$, and since $-K$ is not a fixed component of $\mathscr{F}$, this would imply $\mathscr{F} \cdot K \leqslant-1$. Hence $\mathscr{F}$ is generated by global sections.

Finally, suppose $n=10$. Let $d=\mathscr{F} \cdot \mathscr{E}_{10}$. Then $\mathscr{F}+d K$ is $\mathscr{E}$-excellent (cf. Lemmas 1.4 and 1.6). If $d \geqslant 2$, then $(\mathscr{F}+d K) \cdot K \leqslant-2$ and $\mathscr{F}$ is generated by global sections, as in the case $n \geqslant 11$ treated previously. Only the case $d=1$ remains; by Lemma 1.4, F has the form $-K_{8}-m K_{9}-K_{10}=-(m+2) K_{9}+\left(\mathscr{E}_{9}-\mathscr{E}_{10}\right), m \geqslant 0$.

Now assuming case (b) does not hold, keeping in mind that $j^{*}(\mathscr{F})=0$, it must be that $j^{*}\left(-K_{9}\right) \neq 0$. There are two contingencies: $j^{*}\left(\mathscr{E}_{9}-\mathscr{E}_{10}\right)=0$ and $j^{*}\left(\mathscr{E}_{9}-\mathscr{E}_{10}\right)$ $\neq 0$.

In the former contingency, $\mathscr{E}_{9}-\mathscr{E}_{10}$ is irreducible by Lemma $2.2(2)$, and $j^{*}\left(-(m+2) K_{9}\right)=0$. A calculation using Proposition 1.2(a), (b) shows $h^{0}(V, \mathscr{F})>$ $h^{0}\left(V, \mathscr{F}-\left(\mathscr{E}_{9}-\mathscr{F}_{10}\right)\right)$, so $\mathscr{E}_{9}-\mathscr{E}_{10}$ is not a fixed component of $\mathscr{F}$. Denoting the order of $j^{*}\left(-K_{9}\right)$ in Pic $D$ by $l$, it follows by a previous case that $-l K_{9}$ is generated by global sections, and therefore so is $-(m+2) K_{9}$. Since $\mathscr{F}=\left(\mathscr{E}_{9}-\mathscr{E}_{10}\right)-$ $(m+2) K_{9}$ and $\mathscr{E}_{9}-\mathscr{E}_{10}$ is not a fixed component, $\mathscr{F}$ has no fixed components.

If $\mathscr{F}$ has a base point, it must lie on the global section $N \simeq \mathbf{P}^{1}$ of $\mathscr{E}_{9}-\mathscr{E}_{10}$. Also, since $-(m+2) K_{9}$ is a multiple of $-l K_{9}, \mathscr{F}$ has a base point only if $\mathscr{F}^{\prime}=-l K_{9}+\left(\mathscr{E}_{9}\right.$ $-\mathscr{E}_{10}$ ) does, so it is enough to show that $\mathscr{F}^{\prime}$ has no base points on $N$. A calculation using Proposition 1.2 and $(0.2)$ shows $h^{1}\left(V,-l K_{9}\right)=h^{1}\left(V, \mathscr{F}^{\prime}\right)=1$. Since $\left(\mathscr{E}_{9}-\right.$ $\left.\mathscr{E}_{10}\right) \cdot \mathscr{F}^{\prime} \geqslant 0$, we have $h^{1}\left(N, \mathscr{F}^{\prime} \otimes \mathcal{O}_{N}\right)=0$ by $(0.4)$. Taking cohomology of

$$
0 \rightarrow-l K_{9} \rightarrow \mathscr{F}^{\prime} \rightarrow \mathscr{F}^{\prime} \otimes \mathcal{O}_{N} \rightarrow 0
$$

we conclude that $H^{0}\left(V, \mathscr{F}^{\prime}\right)$ surjects onto $H^{0}\left(N, \mathscr{F}^{\prime} \otimes \mathcal{O}_{N}\right)$. Since $\mathscr{F}^{\prime} \otimes \mathcal{O}_{N}$ has no base points on $N$, neither does $\mathscr{F}^{\prime}$.

Consider now the contingency that $j^{*}\left(\mathscr{E}_{9}-\mathscr{E}_{10}\right) \neq 0$. This implies that $\mathscr{E}_{9}-$ $\mathscr{E}_{10}$ is not effective (Lemma 2.2) and hence that $\mathscr{E}_{9}$ and $\mathscr{E}_{10}$ are irreducible. Denote their unique sections by $E_{9}$ and $E_{10}$, respectively.

We can write $\mathscr{F}$ as $-(m+2) K+\mathscr{E}_{9}+(m+1) \mathscr{E}_{10}$. Clearly, any fixed component of $\mathscr{F}$ is either $E_{9}, E_{10}$, or $D$, and we already showed that $D$ is not a fixed component. But $-K \cdot \mathscr{F}=0$ and $-K \cdot \mathscr{E}_{9}=-K \cdot \mathscr{E}_{10}=1$, so $E_{9}$ and $E_{10}$ cannot be fixed components either, and any base point of $\mathscr{F}$ lies on either $E_{9}$ or $E_{10}$, but off $D$.

Suppose $\mathscr{F}$ has a base point on $\mathscr{E}_{9}$, but off $D$. Writing $\mathscr{F}$ as $-K_{8}-(m+1) K_{10}+$ $m \mathscr{E}_{10}$, we see this means that $-K_{8}$ has a base point on $\mathscr{E}_{9}$. Since $-K_{8} \cdot \mathscr{E}_{9}=0$, it must be that $\mathscr{E}_{9}$ is a fixed component of $-K_{8}$, and thus that $j^{*}\left(-K_{8}-\mathscr{E}_{9}\right)=0$. But $-K_{8}-\mathscr{E}_{9}=-K_{9}$ and we assumed that $j^{*}\left(-K_{9}\right) \neq 0$. Thus $\mathscr{F}$ has no base point on $\mathscr{E}_{9}$. Now writing $\mathscr{F}$ as $-K_{8}-m K_{9}-K_{10}$, we see that if $\mathscr{F}$ has a base point on $E_{10}$ but off $D$, then so does $-K_{8}-m K_{9}$, and since $\left(-K_{8}-m K_{9}\right) \cdot \mathscr{E}_{10}=0, \mathscr{E}_{10}$ must be a fixed component of $-K_{8}-m K_{9}$. But by Lemma 3.3(1), $-K_{10}$ is not a fixed component of $-K_{8}-m K_{9}$. So if $\mathscr{E}_{10}$ is a fixed component of $-K_{8}-m K_{9}$, then

$$
j^{*}\left(-K_{8}-m K_{9}-\mathscr{E}_{10}\right)=0 .
$$

In particular, $j^{*}\left(-K_{9}\right)=j^{*}\left(-K_{10}+\mathscr{E}_{10}\right)=j^{*}\left(-K_{10}-K_{8}-m K_{9}\right)=j^{*}(\mathscr{F})=0$ and assuming that case (b) does not hold, this cannot happen. 
COROLlARY 3.4 Let $\mathscr{F}$ be an effective class with no fixed components. Then (1) any base point of $\mathscr{F}$ lies on the anticanonical divisor $D$ and (2) $\mathscr{F}$ has a base point iff $\mathscr{F} \cdot K=-1$, the base point being where $\mathscr{F}$ meets $D$.

Proof. By Corollary 2.4, if $\mathscr{F}$ is effective and has no fixed components, then $\mathscr{F}$ is almost excellent. If moreover $\mathscr{F} \cdot K=0$, then, as we pointed out in the proof of Theorem $3.1, \mathscr{F}$ is generated by global sections. On the other hand, if $\mathscr{F} \cdot K \leqslant-2$, then again $\mathscr{F}$ is generated by global sections (Lemma 3.3(2)). Therefore, if $\mathscr{F}$ has a base point, it must be that $\mathscr{F} \cdot K=-1$.

Conversely, suppose $\mathscr{F} \cdot K=-1$ and $\mathscr{F}$ is $\mathscr{E}$-excellent for an exceptional configuration $\mathscr{E}=\left\{\mathscr{E}_{0}, \ldots, \mathscr{E}_{n}\right\}$. By Lemma $1.4, \mathscr{F}$ does not lie in Pic $V_{7}$. Thus $\mathscr{F}=a_{0} \mathscr{E}_{0}-$ $a_{1} \mathscr{E}_{1}-\cdots-a_{j} \mathscr{E}_{j}$, where $a_{i}>0$ and $j \geqslant 8$. By Lemma 1.6, $\mathscr{F}^{\prime}=\left(a_{0}-3\right) \mathscr{E}_{0}-$ $\left(a_{1}-1\right) \mathscr{E}_{1}-\cdots-\left(a_{j}-1\right) \mathscr{E}_{j}$ is either trivial or $\mathscr{E}$-excellent, and therefore $h^{1}\left(V, \mathscr{F}^{\prime}\right)=0$. For the purpose of computing cohomology we may assume (Lemma 1.3) that $j=n$, in which case $\mathscr{F}^{\prime}=\mathscr{F}+K$. Taking cohomology of the obvious sequence of line bundles, we have the exact sequence of nontrivial groups

$$
0 \rightarrow H^{0}(V, \mathscr{F}+K) \rightarrow H^{0}(V, \mathscr{F}) \rightarrow H^{0}\left(D, \mathcal{O}_{D} \otimes \mathscr{F}\right) \rightarrow 0 .
$$

But the degree of $\mathcal{O}_{D} \otimes \mathscr{F}$ is unity, so $\mathcal{O}_{D} \otimes \mathscr{F}$ has a base point on $D$, and therefore so does $\mathscr{F}$.

To show that $\mathscr{F}$ has no base point off $D$, we will show that $\mathscr{F}$ is generated by global sections off $D$. By Lemma 1.3 it is enough to show this also in the case that $j=n$. If $n \geqslant 10, \mathscr{F}+K$ is generated by global sections by Lemmas 3.3(2), and 1.6. Thus $\mathscr{F}$ is generated by global sections off $D$. If $n=9$, it follows from Lemma 1.4 that $\mathscr{F}=-K_{8}-m K_{9}, m \geqslant 1$, and from Theorem 3.1(d) that $j^{*}\left(-K_{9}\right) \neq 0$. Thus $-K_{8}$ does not meet $\mathscr{E}_{9}$, and so $\mathscr{F}$ has a base point off $D$ iff $-K_{8}$ does on $V_{8}$. This reduces us to the case $n=8$. But now we have that $\mathscr{F}=-K_{8}$, i.e., $\mathscr{F}$ is the class of $D$, and in particular any base point of $\mathscr{F}$ must be a point of $D$.

\section{BIBLIOGRAPHY}

1. M. Demazure, Surfaces de Del Pezzo, Lecture Notes in Math., Vol. 777, Springer-Verlag, Berlin and New York, 1980, pp. 23-69.

2. P. DuVal, On the Kantor group of a set of points in a plane, Proc. London Math. Soc. 42 (1937), $18-51$.

3. B. Harbourne, Moduli of rational surfaces, Ph.D. Thesis, M.I.T., 1982.

4. R. Hartshorne, Algebraic geometry, Springer-Verlag, Berlin and New York, 1977.

5. E. Looijenga, Rational surfaces with effective anticanonical divisor, Ann. of Math. (2) 114 (1981), 267-322.

6. Y. Manin, Cubic forms: algebra, geometry, arithmetic, North-Holland, Amsterdam, 1974.

7. M. Nagata, On rational surfaces. II, Mem. Coll. Sci. Kyoto (A) 33 (1960), 271-293.

8. On the 14-th problem of Hilbert, Amer. J. Math. 81 (1959), 766-772.

Department of Mathematics, The University of Texas, Austin, TeXas 78712 